\title{
Existence of Nontrivial Solutions for Perturbed Elliptic System in $\mathbb{R}^{N}$
}

\author{
Juan Jiang \\ Department of Mathematics, China University of Mining and Technology, Xuzhou, Jiangsu 221116, China \\ Correspondence should be addressed to Juan Jiang; jiangjuancumt@163.com
}

Received 19 February 2014; Accepted 30 April 2014; Published 13 May 2014

Academic Editor: Shuangjie Peng

Copyright (C) 2014 Juan Jiang. This is an open access article distributed under the Creative Commons Attribution License, which permits unrestricted use, distribution, and reproduction in any medium, provided the original work is properly cited.

We consider the perturbed nonlinear elliptic system $-\varepsilon^{2} \Delta u+V(x) u=K(x)|u|^{2^{*}-2} u+H_{u}(u, v), x \in \mathbb{R}^{N},-\varepsilon^{2} \Delta v+V(x) v=$ $K(x)|v|^{2^{*}-2} v+H_{v}(u, v), x \in \mathbb{R}^{N}$, where $N \geq 3,2^{*}=2 N /(N-2)$ is the Sobolev critical exponent. Under proper conditions on $V, H$, and $K$, the existence result and multiplicity of the system are obtained by using variational method provided $\varepsilon$ is small enough.

\section{Introduction and Main Results}

In this paper, we are concerned with the existence and multiplicity of nontrivial solutions for the following class of elliptic system:

$$
\begin{array}{cc}
-\varepsilon^{2} \Delta u+V(x) u=K(x)|u|^{2^{*}-2} u+H_{u}(u, v), & x \in \mathbb{R}^{N}, \\
-\varepsilon^{2} \Delta v+V(x) v=K(x)|v|^{2^{*}-2} v+H_{v}(u, v), & x \in \mathbb{R}^{N}, \\
u(x), v(x) \longrightarrow 0 \quad \text { as }|x| \longrightarrow \infty, &
\end{array}
$$

where $N \geq 3,2^{*}=2 N /(N-2)$ denotes the Sobolev critical exponent, $V(x)$ is a nonnegative potential, $K(x)$ is bounded positive functions, and $H_{u}(u, v)$ and $H_{v}(u, v)$ are superlinear but subcritical functions.

In the recent years, many papers have considerd the scalar equation

$$
-\varepsilon^{2} \Delta u+V(x) u=g(x, u), \quad x \in \mathbb{R}^{N},
$$

which arises in different models; for example, they are related to the existence of standing waves of the nonlinear Schrödinger equation

$$
i \hbar \frac{\partial \varphi}{\partial t}=-\hbar^{2} \Delta \varphi+(V(x)+E) \varphi-f(\varphi) \quad \forall x \in \mathbb{R}^{N} .
$$

A standing wave of (3) is a solution of the form $\varphi(x, t)=$ $u(x) \exp (-i E t / \hbar)$. We would like to cite the works of Floer and Weinstein [1], Del Pino and Felmer [2, 3], Oh [4], Wang [5], Cingolani and Nolasco [6], Cingolani and Lazzo [7], Ambrosetti et al. [8, 9], Alves and Souto [10, 11], Ding and Lin [12], Liang and Zhang [13], and references therein.

For elliptic systems, we cite the papers of Alves and Soares $[14,15]$, Alves et al. [16], and Ávila and Yang [17].

Motivated by some results found in [12], a natural question arises whether existence of nontrivial solutions continues to hold for the system (1). We study the existence and multiplicity of the nontrivial solutions for the system (1). Our work completes the results obtained in [12], in the sense that we are working with elliptic systems. To prove our main result, we follow some ideas explored in [12] and also use arguments developed in [13, 18].

In this work, we assume the following assumptions:

$\left(V_{0}\right) V \in C\left(\mathbb{R}^{N}, \mathbb{R}\right), V(0)=\inf _{x \in \mathbb{R}^{N}} V(x)=0$, and there is a constant $b>0$ such that the set $\nu^{b}=\left\{x \in \mathbb{R}^{N}\right.$ : $V(x)<b\}$ has finite Lebesgue measure;

$\left(K_{0}\right) K(x) \in C\left(\mathbb{R}^{N}, \mathbb{R}^{+}\right), 0<\inf K \leq \sup K<\infty ;$

$\left(H_{1}\right) H \in C^{1}\left(\mathbb{R}^{+} \times \mathbb{R}^{+}, \mathbb{R}\right), H_{s}(s, t), H_{t}(s, t)=o(1)$ as $(s, t) \rightarrow(0,0)$; 
$\left(H_{2}\right)$ there exist $2<q<2^{*}$ and $c>0$ such that

$$
\left|H_{s}(s, t)\right|,\left|H_{t}(s, t)\right| \leq c\left(1+|s|^{q-1}+|t|^{q-1}\right) ;
$$

$\left(H_{3}\right)$ there exist $a_{0}>0, \alpha, \beta>2, \theta \in\left(2,2^{*}\right)$ such that

$$
\begin{gathered}
H(s, t) \geq a_{0}\left(|s|^{\alpha}+|t|^{\beta}\right), \\
0<\theta H(s, t) \leq s H_{s}(s, t)+t H_{t}(s, t) \quad \forall s>0, t>0 .
\end{gathered}
$$

Our main results are as follows.

Theorem 1. Assume that $\left(V_{0}\right)\left(K_{0}\right)$ and $\left(H_{1}\right)-\left(H_{3}\right)$ hold. Then, for any $\sigma>0$, there exists $\varepsilon_{\sigma}>0$ such that $\varepsilon \leq \varepsilon_{\sigma}$; the perturbed elliptic system (1) has one least energy solution $\left(u_{\varepsilon}, v_{\varepsilon}\right)$ which satisfies

$$
\frac{\theta-2}{2 \theta} \int_{\mathbb{R}^{N}} \varepsilon^{2}\left(\left|\nabla u_{\varepsilon}\right|^{2}+\left|\nabla v_{\varepsilon}\right|^{2}\right)+V(x)\left(\left|u_{\varepsilon}\right|^{2}+\left|v_{\varepsilon}\right|^{2}\right) \leq \sigma \varepsilon^{N} .
$$

Theorem 2. Let $\left(V_{0}\right)\left(K_{0}\right)$ and $\left(H_{1}\right)-\left(H_{3}\right)$ be satisfied. Moreover, assume that $H(u, v)$ is even in $(u, v)$; then, for any $m \in \mathbb{N}$ and $\sigma>0$, there is $\varepsilon_{m \sigma}>0$ such that $\varepsilon \leq \varepsilon_{m \sigma}$; the system (1) has at least $m$ pairs of solutions $\left(u_{\varepsilon}, v_{\varepsilon}\right)$ which satisfy the estimate (6).

The main difficulty in this paper is the lack of compactness of the energy functional associated to the system (1). To overcome this difficulty, we carefully make estimates and prove that there is a Palais-Smale sequence that has a strongly convergent subsequence. The main results in the present paper can be seen as a complement of studies developed in [12].

This paper is organized as follows. In Section 2, we describe some notations and preliminaries. Section 3 is devoted to the behavior of (PS) sequence and the mountain pass level of $J_{\varepsilon}$. Finally, in Section 4, we give the proofs of Theorem 1 and Theorem 2.

\section{Preliminaries}

Let $\lambda=\varepsilon^{-2}$. The system (1) reads then as

$$
\begin{array}{cc}
-\Delta u+\lambda V(x) u=\lambda K(x)|u|^{2^{*}-2} u+\lambda H_{u}(u, v), & x \in \mathbb{R}^{N}, \\
-\Delta v+\lambda V(x) v=\lambda K(x)|v|^{2^{*}-2} v+\lambda H_{v}(u, v), & x \in \mathbb{R}^{N}, \\
u(x), v(x) \longrightarrow 0 \quad \text { as }|x| \longrightarrow \infty . &
\end{array}
$$

We will prove the following result.

Theorem 3. Assume that $\left(V_{0}\right)\left(K_{0}\right)$ and $\left(H_{1}\right)-\left(H_{3}\right)$ hold. Then, for $\sigma>0$, there exists $\Lambda_{\sigma}>0$ such that if $\lambda \geq \Lambda_{\sigma}$, the elliptic system (7) has one positive solution of least energy $\left(u_{\lambda}, v_{\lambda}\right)$ which satisfies

$$
\begin{aligned}
& \frac{\theta-2}{2 \theta} \int_{\mathbb{R}^{N}}\left(\left|\nabla u_{\lambda}\right|^{2}+\left|\nabla v_{\lambda}\right|^{2}+\lambda V(x)\left(\left|u_{\lambda}\right|^{2}+\left|v_{\lambda}\right|^{2}\right)\right) \\
& \leq \sigma \lambda^{1-N / 2} .
\end{aligned}
$$

Theorem 4. Assume that $\left(V_{0}\right)\left(K_{0}\right)$ and $\left(H_{1}\right)-\left(H_{3}\right)$ are satisfied. Moreover, assume that $H(u, v)$ is even in $(u, v)$; then, for any $m \in \mathbb{N}$ and $\sigma>0$, there exists $\Lambda_{\sigma, m}>0$ such that if $\lambda \geq \Lambda_{\sigma, m}$, the system (7) has at least $m$ pairs of solutions $\left(u_{\lambda}, v_{\lambda}\right)$ satisfying the estimate $(8)$.

For the convenience, we quote the necessary notations. The space

$$
E_{\lambda}:=\left\{u \in H^{1}\left(\mathbb{R}^{N}\right): \int_{\mathbb{R}^{N}} \lambda V(x) u^{2}<\infty, \lambda>0\right\}
$$

is a Hilbert space equipped with the inner product

$$
(u, v)_{\lambda}:=\int_{\mathbb{R}^{N}}(\nabla u \nabla v+\lambda V(x) u v)
$$

and the associated norm $\|u\|_{\lambda}^{2}=(u, u)_{\lambda}$. From the assumption $\left(V_{0}\right)$, we conclude that $E_{1}$ embeds continuously in $H^{1}\left(\mathbb{R}^{N}\right)$. Moreover, observe that the norm $\|\cdot\|$ is equivalent to the one $\|\cdot\|_{\lambda}$ for each $\lambda>0$. It is obvious that, for each $p \in\left[2,2^{*}\right]$, there exists $c_{p}>0$ such that if $\lambda \geq 1$

$$
\|u\|_{L^{p}} \leq c_{p}\|u\|_{1} \leq c_{p}\|u\|_{\lambda} \quad \forall u \in E_{1} .
$$

Set $E=E_{\lambda} \times E_{\lambda}$ and $\|(u, v)\|_{E}^{2}=\|u\|_{\lambda}^{2}+\|v\|_{\lambda}^{2}$ for $(u, v) \in E$. The energy functional associated with $(7)$ is defined by

$$
\begin{aligned}
J_{\lambda}(u, v)= & \frac{1}{2} \int_{\mathbb{R}^{N}}\left(|\nabla u|^{2}+\lambda V(x)|u|^{2}+|\nabla v|^{2}+\lambda V(x)|v|^{2}\right) \\
& -\lambda \int_{\mathbb{R}^{N}} \frac{K(x)}{2^{*}}\left(|u|^{2^{*}}+|v|^{2^{*}}\right)-\lambda \int H(u, v) \\
= & \frac{1}{2}\|(u, v)\|_{E}^{2}-\lambda \int_{\mathbb{R}^{N}} G(x, u, v) \quad \text { for }(u, v) \in E,
\end{aligned}
$$

where $G(x, u, v)=\left(K(x) / 2^{*}\right)\left(|u|^{2^{*}}+|v|^{2^{*}}\right)+H(u, v)$.

Under the assumptions of Theorem 3, standard arguments [18] show that $J_{\lambda} \in C^{1}(E, \mathbb{R})$ and the critical points of $J_{\lambda}$ are weak solutions of the elliptic system (7).

\section{Technical Lemmas}

Lemma 5. If $\left\{\left(u_{n}, v_{n}\right)\right\}$ is a $(P S)_{c}$ sequence for $J_{\lambda}$, then $c \geq 0$ and $\left\{\left(u_{n}, v_{n}\right)\right\}$ is bounded in $E$.

Proof. From $\left(K_{0}\right)$ and $\left(H_{3}\right)$, we get

$$
\begin{aligned}
J_{\lambda}\left(u_{n}, v_{n}\right)-\frac{1}{\theta} J_{\lambda}^{\prime}\left(u_{n}, v_{n}\right)\left(u_{n}, v_{n}\right) \\
=\left(\frac{1}{2}-\frac{1}{\theta}\right)\left\|\left(u_{n}, v_{n}\right)\right\|_{E}^{2} \\
\quad+\left(\frac{1}{\theta}-\frac{1}{2^{*}}\right) \lambda \int_{\mathbb{R}^{N}} K(x)\left(\left|u_{n}\right|^{2^{*}}+\left|v_{n}\right|^{2^{*}}\right) \\
\quad+\frac{\lambda}{\theta} \int_{\mathbb{R}^{N}}\left(u_{n} H_{u}\left(u_{n}, v_{n}\right)+v_{n} H_{v}\left(u_{n}, v_{n}\right)-\theta H\left(u_{n}, v_{n}\right)\right) \\
\geq\left(\frac{1}{2}-\frac{1}{\theta}\right)\left\|\left(u_{n}, v_{n}\right)\right\|_{E}^{2} \geq 0 .
\end{aligned}
$$


Together with $J_{\lambda}\left(u_{n}, v_{n}\right) \rightarrow c$ and $J_{\lambda}^{\prime}\left(u_{n}, v_{n}\right) \rightarrow 0$, we have that $\left\{\left(u_{n}, v_{n}\right)\right\}$ is bounded in $E$ and $c \geq 0$.

Lemma 6. Let $d \in\left[2,2^{*}\right)$. There exists a subsequence $\left\{\left(u_{n_{i}}\right.\right.$, $\left.v_{n_{i}}\right)$ \} such that, for any $\varepsilon>0$, there exists $r_{\varepsilon}>0$ with

$$
\limsup _{i \rightarrow \infty} \int_{B_{i} \backslash B_{r}}\left|u_{n_{i}}\right|^{d}+\left|v_{n_{i}}\right|^{d} \leq \varepsilon \quad \forall r \geq r_{\varepsilon}
$$

where $B_{r}:=\left\{x \in \mathbb{R}^{N}:|x| \leq r\right\}$.

Proof. This proof is similar to the one of Lemma 3.2 [12], so we omit it.

Let $\eta \in C^{\infty}\left(\mathbb{R}^{+}\right)$satisfying $0 \leq \eta(t) \leq 1, t \geq 0 . \eta(t)=1$ if $t \leq 1$ and $\eta(t)=0$ if $t \geq 2$. Define $\tilde{u}_{j}(x)=\eta(2|x| / j) u(x)$, $\widetilde{v}_{j}(x)=\eta(2|x| / j) v(x)$; then $\tilde{u}_{j} \rightarrow u, \widetilde{v}_{j} \rightarrow v$ in $E_{\lambda}$.

Lemma 7. One has

$$
\begin{gathered}
\lim _{j \rightarrow \infty} \mid \int_{\mathbb{R}^{N}}\left(H_{s}\left(u_{n_{j}}, v_{n_{j}}\right)-H_{s}\left(u_{n_{j}}-\widetilde{u}_{j}, v_{n_{j}}-\widetilde{v}_{j}\right)\right. \\
\left.-H_{s}\left(\tilde{u}_{j}, \widetilde{v}_{j}\right)\right) \varphi \mid=0, \\
\lim _{j \rightarrow \infty} \mid \int_{\mathbb{R}^{N}}\left(H_{t}\left(u_{n_{j}}, v_{n_{j}}\right)-H_{t}\left(u_{n_{j}}-\tilde{u}_{j}, v_{n_{j}}-\widetilde{v}_{j}\right)\right. \\
\left.-H_{t}\left(\tilde{u}_{j}, \tilde{v}_{j}\right)\right) \psi \mid=0,
\end{gathered}
$$

uniformly in $(\varphi, \psi) \in E$ with $\|(\varphi, \psi)\|_{E} \leq 1$.

Proof. The proof of Lemma 7 is similar to the one of Lemma 3.3 [12], so we omit it.

Lemma 8. One has along a subsequence

$$
\begin{gathered}
J_{\lambda}\left(u_{n}-\tilde{u}_{n}, v_{n}-\widetilde{v}_{n}\right) \longrightarrow c-J_{\lambda}(u, v) \\
J_{\lambda}^{\prime}\left(u_{n}-\tilde{u}_{n}, v_{n}-\widetilde{v}_{n}\right) \longrightarrow 0 \text { in } E^{*} .
\end{gathered}
$$

Proof. Since $\tilde{u}_{j} \rightarrow u, \widetilde{v}_{j} \rightarrow v$ in $E_{\lambda}$ and $\left(u_{j}, v_{j}\right) \rightarrow(u, v)$ in $E$, we have

$$
\begin{aligned}
& J_{\lambda}\left(u_{n}-\tilde{u}_{n}, v_{n}-\tilde{v}_{n}\right) \\
& =J_{\lambda}\left(u_{n}, v_{n}\right)-J_{\lambda}\left(\widetilde{u}_{n}, \widetilde{v}_{n}\right) \\
& \quad+\frac{\lambda}{2^{*}} \int_{\mathbb{R}^{N}} K(x)\left(\left|u_{n}\right|^{2^{*}}-\left|u_{n}-\widetilde{u}_{n}\right|^{2^{*}}-\left|\widetilde{u}_{n}\right|^{2^{*}}\right) \\
& \quad+\frac{\lambda}{2^{*}} \int_{\mathbb{R}^{N}} K(x)\left(\left|v_{n}\right|^{2^{*}}-\left|v_{n}-\widetilde{v}_{n}\right|^{2^{*}}-\left|\widetilde{v}_{n}\right|^{2^{*}}\right) \\
& \quad+\lambda \int_{\mathbb{R}^{N}} H\left(u_{n}, v_{n}\right)-H\left(u_{n}-\widetilde{u}_{n}, v_{n}-\widetilde{v}_{n}\right) \\
& \quad-H\left(\widetilde{u}_{n}, \widetilde{v}_{n}\right)+o(1) .
\end{aligned}
$$

In connection with the proof of Brezis-Lieb lemma, it is easy to check that

$$
\begin{gathered}
\lim _{n \rightarrow \infty} \int_{\mathbb{R}^{N}} K(x)\left(\left|u_{n}\right|^{2^{*}}-\left|u_{n}-\widetilde{u}_{n}\right|^{2^{*}}-\left|\widetilde{u}_{n}\right|^{2^{*}}\right)=0, \\
\lim _{n \rightarrow \infty} \int_{\mathbb{R}^{N}} K(x)\left(\left|v_{n}\right|^{2^{*}}-\left|v_{n}-\widetilde{v}_{n}\right|^{2^{*}}-\left|\widetilde{v}_{n}\right|^{2^{*}}\right)=0, \\
\lim _{n \rightarrow \infty} \int_{\mathbb{R}^{N}} H\left(u_{n}, v_{n}\right)-H\left(u_{n}-\widetilde{u}_{n}, v_{n}-\widetilde{v}_{n}\right)-H\left(\widetilde{u}_{n}, \widetilde{v}_{n}\right)=0 .
\end{gathered}
$$

Note that $J_{\lambda}\left(u_{n}, v_{n}\right) \rightarrow c$ and $J_{\lambda}\left(\widetilde{u}_{n}, \widetilde{v}_{n}\right) \rightarrow J_{\lambda}(u, v)$; we get

$$
J_{\lambda}\left(u_{n}-\tilde{u}_{n}, v_{n}-\widetilde{v}_{n}\right) \longrightarrow c-J_{\lambda}(u, v)
$$

For any $(\varphi, \psi) \in E$, we have

$$
\begin{aligned}
& J_{\lambda}^{\prime}\left(u_{n}-\tilde{u}_{n}, v_{n}-\widetilde{v}_{n}\right)(\varphi, \psi) \\
& =J_{\lambda}^{\prime}\left(u_{n}, v_{n}\right)(\varphi, \psi)-J_{\lambda}^{\prime}\left(\widetilde{u}_{n}, \widetilde{v}_{n}\right)(\varphi, \psi) \\
& +\lambda \int_{\mathbb{R}^{N}} K(x)\left(\left|u_{n}\right|^{2^{*}-2} u_{n}-\left|u_{n}-\widetilde{u}_{n}\right|^{2^{*}-2}\left(u_{n}-\widetilde{u}_{n}\right)\right. \\
& \left.\quad-\left|\widetilde{u}_{n}\right|^{2^{*}-2} \widetilde{u}_{n}\right) \varphi \\
& +\lambda \int_{\mathbb{R}^{N}} K(x)\left(\left|v_{n}\right|^{2^{*}-2} v_{n}-\left|v_{n}-\widetilde{v}_{n}\right|^{2^{*}-2}\left(v_{n}-\widetilde{v}_{n}\right)\right. \\
& \left.\quad-\left|\widetilde{v}_{n}\right|^{2^{*}-2} \widetilde{v}_{n}\right) \psi \\
& +\lambda \int_{\mathbb{R}^{N}}\left(H_{u}\left(u_{n}, v_{n}\right)-H_{u}\left(u_{n}-\widetilde{u}_{n}, v_{n}-\widetilde{v}_{n}\right)\right. \\
& \left.-H_{u}\left(\widetilde{u}_{n}, \widetilde{v}_{n}\right)\right) \varphi \\
& +\lambda \int_{\mathbb{R}^{N}}\left(H_{v}\left(u_{n}, v_{n}\right)-H_{v}\left(u_{n}-\tilde{u}_{n}, v_{n}-\widetilde{v}_{n}\right)\right. \\
& \left.-H_{v}\left(\widetilde{u}_{n}, \widetilde{v}_{n}\right)\right) \psi .
\end{aligned}
$$

It is standard to check that

$$
\begin{gathered}
\lim _{n \rightarrow \infty} \int_{\mathbb{R}^{N}} K(x)\left(\left|u_{n}\right|^{2^{*}-2} u_{n}-\left|u_{n}-\widetilde{u}_{n}\right|^{2^{*}-2}\left(u_{n}-\widetilde{u}_{n}\right)\right. \\
\left.-\left|\widetilde{u}_{n}\right|^{2^{*}-2} \widetilde{u}_{n}\right) \varphi=0, \\
\lim _{n \rightarrow \infty} \int_{\mathbb{R}^{N}} K(x)\left(\left|v_{n}\right|^{2^{*}-2} v_{n}-\left|v_{n}-\widetilde{v}_{n}\right|^{2^{*}-2}\left(v_{n}-\widetilde{v}_{n}\right)\right. \\
\left.-\left|\widetilde{v}_{n}\right|^{2^{*}-2} \widetilde{v}_{n}\right) \psi=0,
\end{gathered}
$$

uniformly in $\|(\varphi, \psi)\|_{E} \leq 1$. Together with Lemma 7, we complete the proof of Lemma 8 . 
Let $u_{n}^{1}=u_{n}-\widetilde{u}_{n}, v_{n}^{1}=v_{n}-\widetilde{v}_{n}$; then $\left(u_{n}, v_{n}\right) \rightarrow(u, v)$ in $E$ if and only if $\left(u_{n}^{1}, v_{n}^{1}\right) \rightarrow(0,0)$ in $E$. Observe that

$$
\begin{aligned}
J_{\lambda}\left(u_{n}^{1}, v_{n}^{1}\right)-\frac{1}{2} J_{\lambda}^{\prime}\left(u_{n}^{1}, v_{n}^{1}\right)\left(u_{n}^{1}, v_{n}^{1}\right) \\
=\left(\frac{1}{2}-\frac{1}{2^{*}}\right) \lambda \int_{\mathbb{R}^{N}} K(x)\left(\left|u_{n}^{1}\right|^{2^{*}}+\left|v_{n}^{1}\right|^{2^{*}}\right) \\
\quad+\lambda \int_{\mathbb{R}^{N}}\left(\frac{1}{2}\left(u_{n}^{1} H_{u}\left(u_{n}^{1}, v_{n}^{1}\right)+v_{n}^{1} H_{v}\left(u_{n}^{1}, v_{n}^{1}\right)\right)\right. \\
\left.\quad-H\left(u_{n}^{1}, v_{n}^{1}\right)\right) \\
\geq \frac{\lambda}{N} K_{\min } \int_{\mathbb{R}^{N}}\left(\left|u_{n}^{1}\right|^{2^{*}}+\left|v_{n}^{1}\right|^{2^{*}}\right)
\end{aligned}
$$

where $K_{\min }=\inf _{x \in \mathbb{R}^{N}} K(x)>0$. By Lemma 8, we get

$$
\left\|u_{n}^{1}\right\|_{L^{2^{*}}}^{2^{*}}+\left\|v_{n}^{1}\right\|_{L^{2^{*}}}^{2^{*}} \leq \frac{N\left(c-J_{\lambda}(u, v)\right)}{\lambda K_{\min }}+o(1) .
$$

Meanwhile, by $\left(H_{2}\right)$ and $\left(H_{3}\right)$, there exists $C_{b}>0$ such that

$$
\begin{gathered}
\int_{\mathbb{R}^{N}} K(x)\left(|u|^{2^{*}}+|v|^{2^{*}}\right)+u H_{s}(u, v)+v H_{t}(u, v) \\
\leq b\left(\|u\|_{L^{2}}^{2}+\|v\|_{L^{2}}^{2}\right)+C_{b}\left(\|u\|_{L^{2^{*}}}^{2^{*}}+\|v\|_{L^{2^{*}}}^{2^{*}}\right) .
\end{gathered}
$$

Lemma 9. There is a constant $\alpha_{0}>0$ (independent of $\lambda$ ) such that, for any $(P S)_{c}$ sequence $\left\{\left(u_{n}, v_{n}\right)\right\} \subset E$ for $J_{\lambda}$ with $\left(u_{n}, v_{n}\right) \rightarrow(u, v)$, either $\left(u_{n}, v_{n}\right) \rightarrow(u, v)$ or $c-J_{\lambda}(u, v) \geq$ $\alpha_{0} \lambda^{1-N / 2}$.

Proof. On the contrary, if $\left(u_{n}, v_{n}\right) \nrightarrow(u, v)$, we have

$$
\liminf _{n \rightarrow \infty}\left\|\left(u_{n}^{1}, v_{n}^{1}\right)\right\|_{E}>0, \quad c-J_{\lambda}(u, v)>0 .
$$

Let $V_{b}(x)=\max \{V(x), b\}$, where $b$ is the positive constant in the assumption $\left(V_{0}\right)$. In connection with the set $\nu^{b}$ which has finite measure and $u_{n}^{1}, v_{n}^{1} \rightarrow 0$ in $L_{\text {loc }}^{2}\left(\mathbb{R}^{N}\right)$, we have

$$
\int_{\mathbb{R}^{N}} V(x)\left(\left|u_{n}^{1}\right|^{2}+\left|v_{n}^{1}\right|^{2}\right)=\int_{\mathbb{R}^{N}} V_{b}(x)\left(\left|u_{n}^{1}\right|^{2}+\left|v_{n}^{1}\right|^{2}\right)+o(1) .
$$

Therefore, we obtain

$$
\begin{aligned}
& S\left(\left\|u_{n}^{1}\right\|_{L^{2^{*}}}^{2}+\left\|v_{n}^{1}\right\|_{L^{2^{*}}}^{2}\right) \\
& \leq \int_{\mathbb{R}^{N}}\left(\left|\nabla u_{n}^{1}\right|^{2}+\left|\nabla v_{n}^{1}\right|^{2}+\lambda V(x)\left(\left|u_{n}^{1}\right|^{2}+\left|v_{n}^{1}\right|^{2}\right)\right) \\
& \quad-\int_{\mathbb{R}^{N}} \lambda V(x)\left(\left|u_{n}^{1}\right|^{2}+\left|v_{n}^{1}\right|^{2}\right) \\
& \leq \lambda \int_{\mathbb{R}^{N}} K(x)\left(\left|u_{n}^{1}\right|^{2^{*}}+\left|v_{n}^{1}\right|^{2^{*}}\right) \\
& \quad+\int_{\mathbb{R}^{N}} \lambda V_{b}(x)\left(\left|u_{n}^{1}\right|^{2}+\left|v_{n}^{1}\right|^{2}\right)+o(1) \\
& \leq \lambda C_{b}\left(\| u_{n}^{1}, v_{n}^{1}\right)+v_{n}^{1} H_{t}^{2^{*}}\left(u_{n}^{1}, v_{n}^{1}\right)
\end{aligned}
$$

where $S$ is the best Sobolev constant which satisfies

$$
S\|u\|_{L^{2^{*}}}^{2} \leq \int_{\mathbb{R}^{N}}|\nabla u|^{2} \quad \forall u \in H^{1}\left(\mathbb{R}^{N}\right) .
$$

By (23), we get

$$
\begin{aligned}
S & \leq \lambda C_{b}\left(\left\|u_{n}^{1}\right\|_{L^{2^{*}}}^{2^{*}}+\left\|v_{n}^{1}\right\|_{L^{2^{*}}}^{2^{*}}\right)^{\left(2^{*}-2\right) / 2^{*}}+o(1) \\
& \leq \lambda C_{b}\left(\frac{N\left(c-J_{\lambda}(u, v)\right)}{\lambda K_{\min }}\right)^{2 / N}+o(1) \\
& =\lambda^{1-2 / N} C_{b}\left(\frac{N}{K_{\min }}\right)^{2 / N}\left(c-J_{\lambda}(u, v)\right)^{2 / N}+o(1) .
\end{aligned}
$$

Therefore, $\alpha_{0} \lambda^{1-N / 2} \leq c-J_{\lambda}(u, v)+o(1)$, where $\alpha_{0}=$ $S^{N / 2} C_{b}^{-N / 2} N^{-1} K_{\min }$. The proof is completed.

Lemma 10. There is a constant $\alpha_{0}>0$ (independent of $\lambda$ ) such that if a sequence $\left\{\left(u_{n}, v_{n}\right)\right\} \subset E$ satisfies

$$
\begin{gathered}
J_{\lambda}\left(u_{n}, v_{n}\right) \longrightarrow c<\alpha_{0} \lambda^{1-N / 2}, \\
J_{\lambda}^{\prime}\left(u_{n}, v_{n}\right) \longrightarrow 0 \text { in } E^{*},
\end{gathered}
$$

then $\left\{\left(u_{n}, v_{n}\right)\right\}$ is relatively compact in $E$.

Proof. Lemma 9 implies that $J_{\lambda}$ satisfies the following local $(\mathrm{PS})_{c}$ condition. The proof is completed.

Next, we consider $\lambda \geq 1$ and see that $J_{\lambda}$ has the mountain pass structure.

Lemma 11. Assume that $\left(V_{0}\right),\left(K_{0}\right)$, and $\left(H_{1}\right)-\left(H_{3}\right)$ hold. There exist $\alpha_{\lambda}, \rho_{\lambda}>0$ such that

$$
\begin{aligned}
& J_{\lambda}(u, v)>0, \quad 0<\|(u, v)\|_{E}<\rho_{\lambda} \\
& J_{\lambda}(u, v) \geq \alpha_{\lambda}, \quad \text { if }\|(u, v)\|_{E}=\rho_{\lambda} .
\end{aligned}
$$

Proof. Observe that $\|u\|_{L^{2}}^{2}+\|v\|_{L^{2}}^{2} \leq C_{1}\|(u, v)\|_{E}^{2}$. For $\delta \leq$ $\left(4 \lambda C_{1}\right)^{-1}$, there is a constant $C_{\delta}$ such that

$$
J_{\lambda}(u, v) \geq \frac{1}{4}\|(u, v)\|_{E}^{2}-\lambda C_{\delta}\left(\|u\|_{L^{2^{*}}}^{2^{*}}+\|v\|_{L^{2^{*}}}^{2^{*}}\right),
$$

which implies that the required conclusions hold.

Lemma 12. Under the assumptions of Theorem 3, for any finite dimensional subspace $F \subset E$, we get

$$
J_{\lambda}(u, v) \longrightarrow-\infty \quad \text { as }(u, v) \in F,\|(u, v)\|_{E} \longrightarrow \infty .
$$

Proof. By $\left(K_{0}\right)$ and $\left(H_{3}\right)$, we get

$$
J_{\lambda}(u, v) \leq \frac{1}{2}\|(u, v)\|_{E}^{2}-\lambda a_{0}\left(\|u\|_{L^{\alpha}}^{\alpha}+\|v\|_{L^{\beta}}^{\beta}\right) \quad \forall(u, v) \in E .
$$

Since all norms in a finite dimensional space are equivalent and $\alpha, \beta>2$, we easily obtain the desired conclusion. 
Define the functional

$$
\begin{aligned}
\Phi_{\lambda}(u, v)= & \frac{1}{2} \int_{\mathbb{R}^{N}}\left(|\nabla u|^{2}+|\nabla v|^{2}+\lambda V(x)\left(|u|^{2}+|v|^{2}\right)\right) \\
& -a_{0} \lambda \int_{\mathbb{R}^{N}}\left(|u|^{\alpha}+|v|^{\beta}\right), \\
I_{\lambda}(u, v)= & \frac{1}{2} \int_{\mathbb{R}^{N}}\left(|\nabla u|^{2}+|\nabla v|^{2}+V\left(\lambda^{-1 / 2} x\right)\left(|u|^{2}+|v|^{2}\right)\right) \\
& -a_{0} \int_{\mathbb{R}^{N}}\left(|u|^{\alpha}+|v|^{\beta}\right) .
\end{aligned}
$$

The standard arguments show that $\Phi_{\lambda} \in C^{1}(E)$ and $J_{\lambda}(u, v) \leq$ $\Phi_{\lambda}(u, v),(u, v) \in E$.

Observe that

$$
\begin{aligned}
& \inf \left\{\int_{\mathbb{R}^{N}}|\nabla \phi|^{2}: \phi \in C_{0}^{\infty}\left(\mathbb{R}^{N}, \mathbb{R}\right),\|\phi\|_{L^{\alpha}}=1\right\}=0, \\
& \inf \left\{\int_{\mathbb{R}^{N}}|\nabla \psi|^{2}: \psi \in C_{0}^{\infty}\left(\mathbb{R}^{N}, \mathbb{R}\right),\|\psi\|_{L^{\beta}}=1\right\}=0 .
\end{aligned}
$$

For any $\delta>0$, there are $\phi_{\delta}, \psi_{\delta} \in C_{0}^{\infty}\left(\mathbb{R}^{N}, \mathbb{R}\right)$ with $\left\|\phi_{\delta}\right\|_{L^{\alpha}}=$ $\left\|\psi_{\delta}\right\|_{L^{\beta}}=1$ and $\operatorname{supp}\left(\phi_{\delta}, \psi_{\delta}\right) \subset B_{r_{\delta}}(0)$ such that $\left\|\nabla \phi_{\delta}\right\|_{L^{2}}^{2}$, $\left\|\nabla \psi_{\delta}\right\|_{L^{2}}^{2}<\delta$. Set $e_{\lambda}(x)=\left(\phi_{\delta}(\sqrt{\lambda} x), \psi_{\delta}(\sqrt{\lambda} x)\right)$; then, supp $e_{\lambda} \subset B_{\lambda^{-1 / 2} r_{\delta}}(0)$. For $t \geq 0$, we get

$$
\Phi_{\lambda}\left(t e_{\lambda}\right)=\lambda^{1-N / 2} I_{\lambda}\left(t \phi_{\delta}, t \psi_{\delta}\right)
$$

It is obvious that

$$
\begin{aligned}
\max _{t \geq 0} & I_{\lambda}\left(t \phi_{\delta}, t \psi_{\delta}\right) \\
\leq & \frac{\alpha-2}{2 \alpha\left(\alpha a_{0}\right)^{2 /(\alpha-2)}}\left\{\int_{\mathbb{R}^{N}}\left|\nabla \phi_{\delta}\right|^{2}+V\left(\lambda^{-1 / 2} x\right)\left|\phi_{\delta}\right|^{2}\right\}^{\alpha /(\alpha-2)} \\
& +\frac{\beta-2}{2 \beta\left(\beta a_{0}\right)^{2 /(\beta-2)}} \\
& \times\left\{\int_{\mathbb{R}^{N}}\left|\nabla \psi_{\delta}\right|^{2}+V\left(\lambda^{-1 / 2} x\right)\left|\psi_{\delta}\right|^{2}\right\}^{\beta /(\beta-2)} .
\end{aligned}
$$

In connection with $V(0)=0$ and $\operatorname{supp} \phi_{\delta}$, supp $\psi_{\delta} \subset B_{r_{\delta}}(0)$, there is $\Lambda_{\delta}>0$ such that

$$
\begin{aligned}
& \max _{t \geq 0} J_{\lambda}\left(t \phi_{\delta}, t \psi_{\delta}\right) \\
& \leq \lambda^{1-N / 2}\left(\frac{\alpha-2}{2 \alpha\left(\alpha a_{0}\right)^{2 /(\alpha-2)}}(2 \delta)^{\alpha /(\alpha-2)}\right. \\
&\left.+\frac{\beta-2}{2 \beta\left(\beta a_{0}\right)^{2 /(\beta-2)}}(2 \delta)^{\beta /(\beta-2)}\right),
\end{aligned}
$$

for all $\lambda \geq \Lambda_{\sigma}$.

From (39), we easily obtain the following result.
Lemma 13. For any $\sigma>0$, there exists $\Lambda_{\sigma}>0$ such that, for each $\lambda \geq \Lambda_{\sigma}$, there exists $\bar{e}_{\lambda} \in E$ with $\left\|\bar{e}_{\lambda}\right\|_{E}>\rho_{\lambda}$; we get

$$
J_{\lambda}\left(\bar{e}_{\lambda}\right) \leq 0, \quad \max _{t \geq 0} J_{\lambda}\left(t \bar{e}_{\lambda}\right) \leq \sigma \lambda^{1-N / 2}
$$

where $\rho_{\lambda}$ is defined form Lemma 11.

Proof. This proof is similar to Lemma 4.3 in [12]; it can be easily obtained.

For any $m \in \mathbb{N}$, we choose $m$ functions $\phi_{\delta}^{i} \in C_{0}^{\infty}\left(\mathbb{R}^{N}\right)$ such that $\operatorname{supp} \phi_{\delta}^{i} \cap \operatorname{supp} \phi_{\delta}^{j}=\emptyset, i \neq j,\left\|\phi_{\delta}^{i}\right\|_{L^{\alpha}}=1$, and $\left\|\nabla \phi_{\delta}^{i}\right\|_{L^{2}}^{2}<\delta$. Similarly, one can also get $m$ functions $\psi_{\delta}^{i} \epsilon$ $C_{0}^{\infty}\left(\mathbb{R}^{N}\right)$ with supp $\psi_{\delta}^{i} \cap \operatorname{supp} \psi_{\delta}^{j}=\emptyset, i \neq j,\left\|\psi_{\delta}^{i}\right\|_{L^{\beta}}=1$, and $\left\|\nabla \psi_{\delta}^{i}\right\|_{L^{2}}^{2}<\delta$. Let $r_{\delta}^{m}>0$ such that $\operatorname{supp}\left(\phi_{\delta}^{i}, \psi_{\delta}^{i}\right) \subset B_{r_{\delta}^{m}}^{i}(0)$ for $i=1,2, \ldots, m$. Set $e_{\lambda}^{i}(x)=\left(\phi_{\delta}^{i}(\sqrt{\lambda} x), \psi_{\delta}^{i}(\sqrt{\lambda} x)\right)=\left(f_{\lambda}^{i}, g_{\lambda}^{i}\right)$, $i=1,2, \ldots, m$; then $\operatorname{supp} e_{\lambda}^{i}(x) \subset B_{\lambda^{-1 / 2} r_{\delta}^{m}}(0)$.

Define $F_{\lambda \delta}^{m}=\operatorname{span}\left\{e_{\lambda}^{1}, e_{\lambda}^{2}, \ldots, e_{\lambda}^{m}\right\}$. For each $(u, v)=$ $\sum_{i=1}^{m} k_{i} e_{\lambda}^{i} \in F_{\lambda \delta}^{m}$, we easily get

$$
\begin{aligned}
& J_{\lambda}(u, v)=\sum_{i=1}^{m} J_{\lambda}\left(k_{i} e_{\lambda}^{i}\right), \\
& J_{\lambda}\left(k_{i} e_{\lambda}^{i}\right) \leq \phi_{\lambda}\left(k_{i} e_{\lambda}^{i}\right) .
\end{aligned}
$$

Set $\beta_{\delta}:=\max \left\{\left\|\left(\phi_{\delta}^{i}, \psi_{\delta}^{i}\right)\right\|_{2}^{2}: i=1,2, \ldots, m\right\}$ and choose some $\Lambda_{m \delta}>0$ such that

$$
V\left(\lambda^{-1 / 2} x\right) \leq \frac{\delta}{\beta_{\delta}} \quad \forall|x| \leq r_{\delta}^{m}, \lambda \geq \Lambda_{m \delta}
$$

Furthermore, we have the following inequality:

$$
\begin{aligned}
& \max _{(u, v) \in F_{\lambda \delta}^{m}} J_{\lambda}(u, v) \\
& \leq \lambda^{1-N / 2}\left(\frac{m(\alpha-2)}{2 \alpha\left(\alpha a_{0}\right)^{2 /(\alpha-2)}}(2 \delta)^{\alpha /(\alpha-2)}\right. \\
&\left.+\frac{m(\beta-2)}{2 \beta\left(\beta a_{0}\right)^{2 /(\beta-2)}}(2 \delta)^{\beta /(\beta-2)}\right) .
\end{aligned}
$$

Lemma 14. Under the assumptions of Theorem 4, for any $m \in$ $\mathbb{N}$ and $\sigma>0$, there is $\Lambda_{m \sigma}>0$ such that, for each $\lambda \geq \Lambda_{m \delta}$, we can get an $m$-dimensional subspace $F$ which satisfies

$$
\max _{(u, v) \in F} J_{\lambda}(u, v) \leq \sigma \lambda^{(2-N) / 2} .
$$

Proof. For any $\sigma>0$, we choose $\delta>0$ so small that

$$
\begin{aligned}
& \left(\frac{m(\alpha-2)}{2 \alpha\left(\alpha a_{0}\right)^{2 /(\alpha-2)}}(2 \delta)^{\alpha /(\alpha-2)}\right. \\
& \left.\quad+\frac{m(\beta-2)}{2 \beta\left(\beta a_{0}\right)^{2 /(\beta-2)}}(2 \delta)^{\beta /(\beta-2)}\right) \leq \sigma .
\end{aligned}
$$

Meanwhile, we take $F=F_{\lambda \delta}^{m}$. By (43), we prove the required conclusion. 


\section{Proof of the Main Results}

Proof of Theorem 3. Define $c_{\lambda}=\inf _{\gamma \in \Gamma_{\lambda}} \max _{t \in[0,1]} J_{\lambda}(\gamma(t))$, where $\Gamma_{\lambda}=\left\{\gamma \in C([0,1], E): \gamma(0)=0, \gamma(1)=\bar{e}_{\lambda}\right\}$. For any $0<\sigma<\alpha_{0}$, there exists $\Lambda_{\sigma}>0$ such that, for each $\lambda \geq \Lambda_{\sigma}$, we can choose $c_{\lambda}$ satisfying $c_{\lambda} \leq \sigma \lambda^{1-(N / 2)}$.

By Lemma $9, J_{\lambda}$ satisfies the (PS $)_{\mathcal{C}_{\lambda}}$ condition. Hence, by the mountain pass theorem, there exists $\left(u_{\lambda}, v_{\lambda}\right) \in E$ satisfying $J_{\lambda}^{\prime}\left(u_{\lambda}, v_{\lambda}\right)=0$ and $J_{\lambda}\left(u_{\lambda}, v_{\lambda}\right)=c_{\lambda}$. So $\left(u_{\lambda}, v_{\lambda}\right)$ is a weak solution of (7).

Furthermore, we have

$$
\begin{aligned}
J_{\lambda}\left(u_{\lambda}, v_{\lambda}\right) & =J_{\lambda}\left(u_{\lambda}, v_{\lambda}\right)-\frac{1}{\theta} J_{\lambda}^{\prime}\left(u_{\lambda}, v_{\lambda}\right)\left(u_{\lambda}, v_{\lambda}\right) \\
& \geq\left(\frac{1}{2}-\frac{1}{\theta}\right)\left\|\left(u_{\lambda}, v_{\lambda}\right)\right\|_{E}^{2} .
\end{aligned}
$$

This shows that

$$
\begin{aligned}
& \frac{\theta-2}{2 \theta} \int_{\mathbb{R}^{N}}\left(\left|\nabla u_{\lambda}\right|^{2}+\left|\nabla v_{\lambda}\right|^{2}+\lambda V(x)\left(\left|u_{\lambda}\right|^{2}+\left|v_{\lambda}\right|^{2}\right)\right) \\
& \leq \sigma \lambda^{1-N / 2} .
\end{aligned}
$$

Proof of Theorem 4. By Lemma 14, for any $m \in \mathbb{N}$ and $\sigma \leq$ $\alpha_{0}$, there exists $\Lambda_{m \sigma}$ such that, for $\lambda \geq \Lambda_{m \sigma}$, we choose an $m$-dimensional subspace $F$ with $\max J_{\lambda}(F) \leq \sigma \lambda^{1-N / 2}$. By Lemma 12, there is $R>0$ such that $J_{\lambda}(u) \leq 0$ for all $u \in F \mid B_{R}$. Define $e_{\lambda_{j}}=\inf _{i(\Omega) \geq j} \sup _{u, v \in \Omega} J_{\lambda}(u, v), 1 \leq j \leq m$, where $i(\Omega)$ is a version of Benci's pseudoindex [19].

Since $J_{\lambda}(u, v) \geq \alpha_{\lambda}$ for all $(u, v) \in \partial B_{\rho_{\lambda}}$ (see Lemma 11) and $i(F)=\operatorname{dim} F=m$, one has

$$
\alpha_{\lambda} \leq c_{\lambda_{1}} \leq c_{\lambda_{2}} \leq \cdots \leq c_{\lambda_{m}} \leq \sup _{(u, v) \in F_{\lambda \sigma}^{m}} J_{\lambda}(u, v) \leq \sigma \lambda^{1-N / 2} .
$$

We easily get that $c_{\lambda_{j}}$ are critical levels and $J_{\lambda}$ has at least $m$ pairs of nontrivial critical points. This proof is completed.

\section{Conflict of Interests}

The author declares that there is no conflict of interests regarding the publication of this paper.

\section{Acknowledgments}

The author would like to thank the anonymous referee for precious comments and suggestions about the original paper. This research was supported by the National Natural Science Foundation of China (11271364).

\section{References}

[1] A. Floer and A. Weinstein, "Nonspreading wave packets for the cubic Schrödinger equation with a bounded potential," Journal of Functional Analysis, vol. 69, no. 3, pp. 397-408, 1986.

[2] M. del Pino and P. Felmer, "Multi-peak bound states for nonlinear Schrödinger equations," Journal of Functional Analysis, vol. 149, pp. 245-265, 1997.
[3] M. del Pino and P. Felmer, "Semi-classical states for nonlinear Schrödinger equations," Annales de l'Institut Henri Poincaré, vol. 15, pp. 127-149, 1998.

[4] Y. G. Oh, "On positive multi-bump bound states of nonlinear Schrödinger equations under multiple well potential," Communications in Mathematical Physics, vol. 131, pp. 223-253, 1990.

[5] F. Wang, "On an electromagnetic Schrödinger equation with critical growth," Nonlinear Analysis: Theory, Methods and Applications, vol. 69, no. 11, pp. 4088-4098, 2008.

[6] S. Cingolani and M. Nolasco, "Multi-peak periodic semiclassical states for a class of nonlinear Schrödinger equations," Proceedings of the Royal Society of Edinburgh, vol. 128, no. 6, pp. 1249-1260, 1998.

[7] S. Cingolani and M. Lazzo, "Multiple positive solutions to nonlinear Schrödinger equations with competing potential functions," Journal of Differential Equations, vol. 160, no. 1, pp. 118$138,2000$.

[8] A. Ambrosetti, M. Badiale, and S. Cingolani, "Semiclassical states of nonlinear Schrödinger equations," Archive for Rational Mechanics and Analysis, vol. 140, pp. 285-300, 1997.

[9] A. Ambrosetti, A. Malchiodi, and S. Secchi, "Multiplicity results for some nonlinear Schrödinger equations with potentials," Archive for Rational Mechanics and Analysis, vol. 159, no. 3, pp. 253-271, 2001.

[10] C. O. Alves and M. A. S. Souto, "Local mountain-pass for a class of elliptic problems in $\mathbb{R}^{N}$ involving critical growth," Nonlinear Analysis: Theory, Methods and Applications, vol. 46, no. 4, pp. 495-510, 2001.

[11] C. O. Alves and M. A. S. Souto, "On existence and concentration behavior of ground state solutions for a class of problems with critical growth," Communications on Pure and Applied Mathematics, vol. 3, pp. 417-431, 2002.

[12] Y. H. Ding and F. H. Lin, "Solutions of perturbed Schrödinger equations with critical nonlinearity," Calculus of Variations and Partial Differential Equations, vol. 30, pp. 231-249, 2007.

[13] S. H. Liang and J. H. Zhang, "Solutions of perturbed Schrödinger equations with electromagnetic fields and critical nonlinearity," Proceedings of the Edinburgh Mathematical Society, vol. 54, pp. 131-147, 2011.

[14] C. O. Alves and S. H. M. Soares, "Existence and concentration of positive solutions for a class of gradient systems," Nonlinear Differential Equations and Applications, vol. 12, no. 4, pp. 437456, 2006.

[15] C. O. Alves and S. H. M. Soares, "Existence of solution for a class of quasilinear systems," Advanced Nonlinear Studies, vol. 9, no. 3, pp. 537-564, 2009.

[16] C. O. Alves, S. H. M. Soares, and J. Yang, "On the existence and concentration of solutions for a class Hamiltonian systems in $\mathbb{R}^{N}$," Advanced Nonlinear Studies, vol. 2, pp. 161-180, 2003.

[17] A. I. Ávila and J. Yang, "On the existence and shape of least energy solutions for some elliptic systems," Journal of Differential Equations, vol. 191, no. 2, pp. 348-376, 2003.

[18] J. Mawhin and M. Willem, Critical Point Theory and Hamiltonian Systems, Springer, New York, NY, USA, 1989.

[19] V. Benci, "On critical point theory of indefinite functions in the presence of symmetries," Transactions of the American Mathematical Society, vol. 274, pp. 533-572, 1982. 


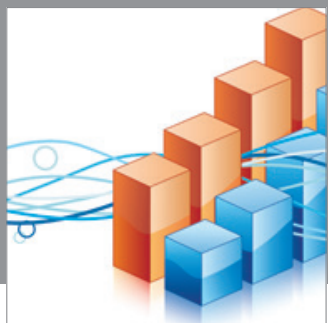

Advances in

Operations Research

mansans

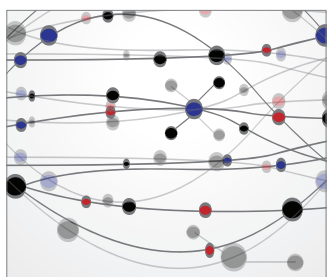

The Scientific World Journal
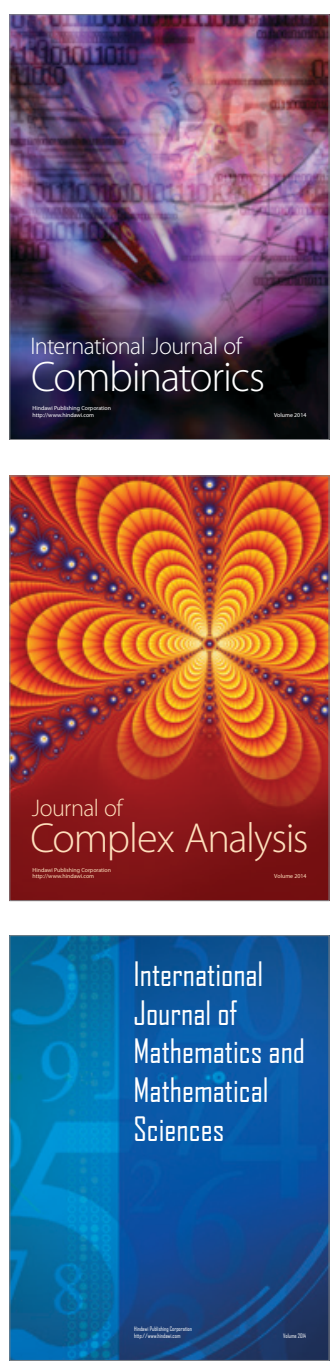
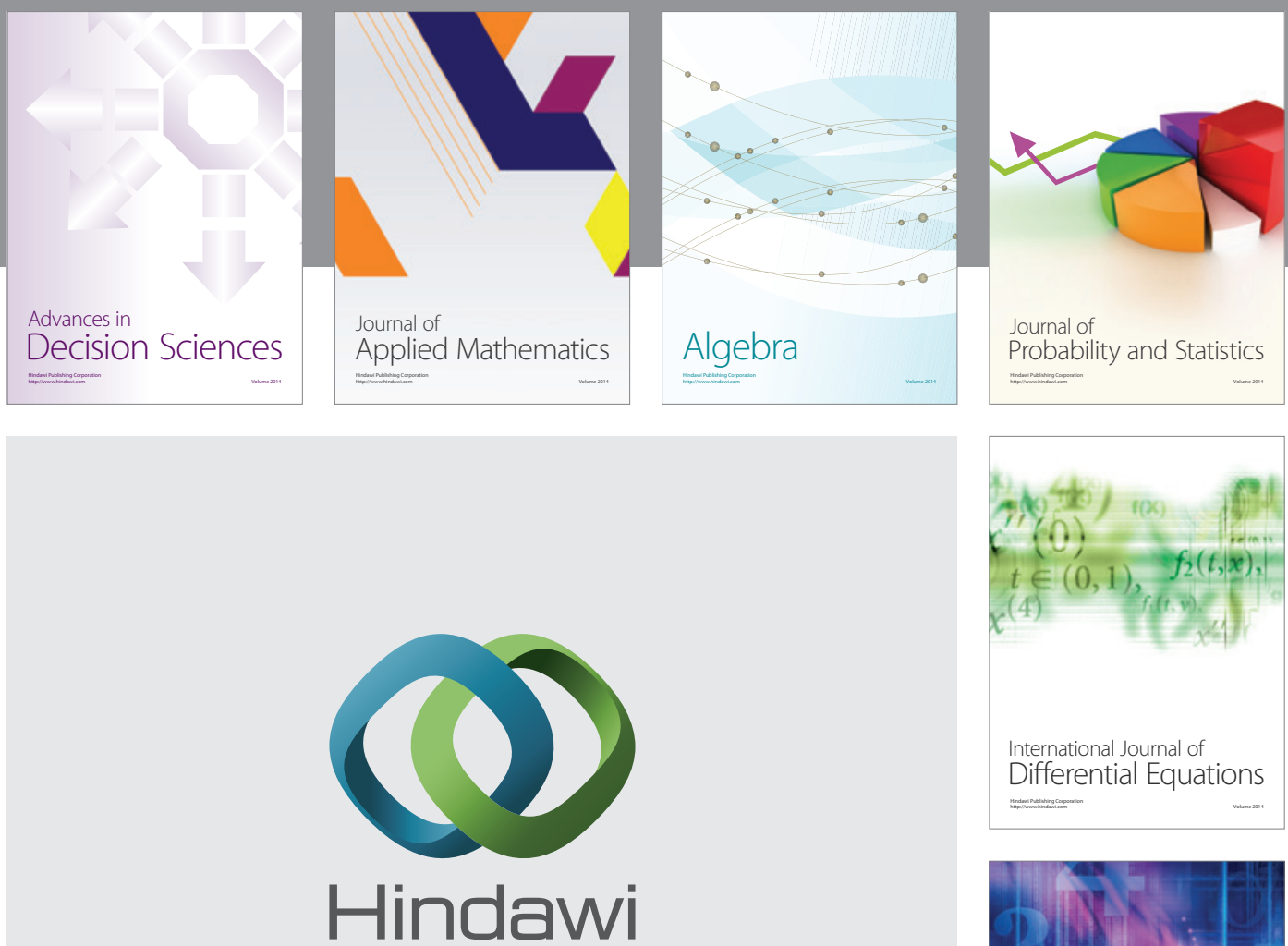

Submit your manuscripts at http://www.hindawi.com
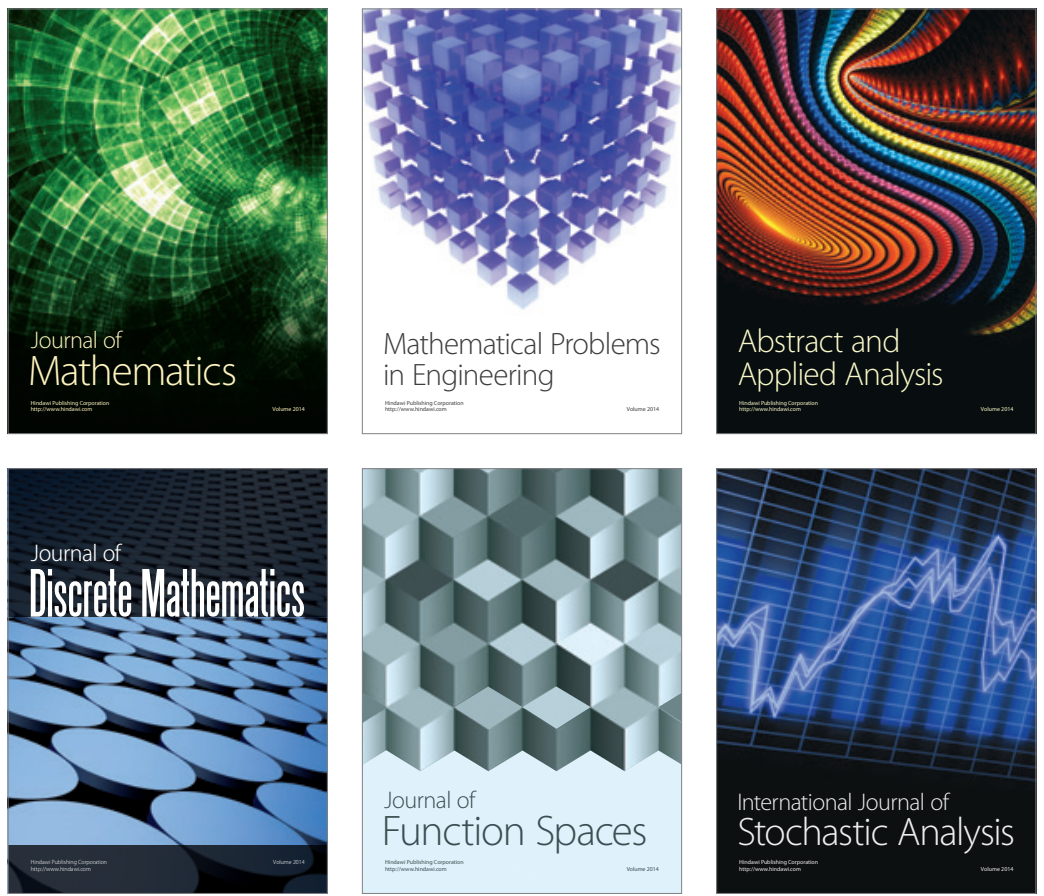

Journal of

Function Spaces

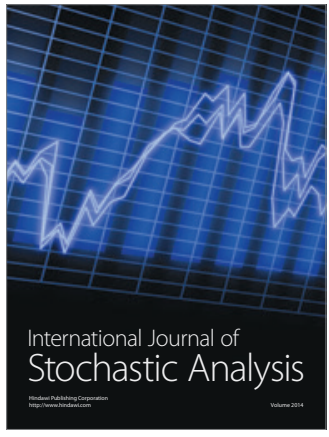

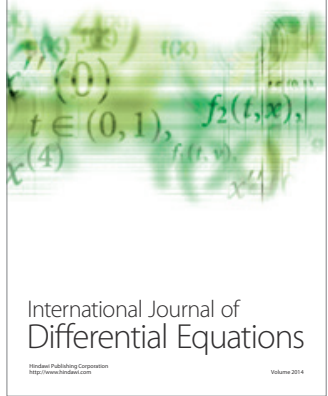
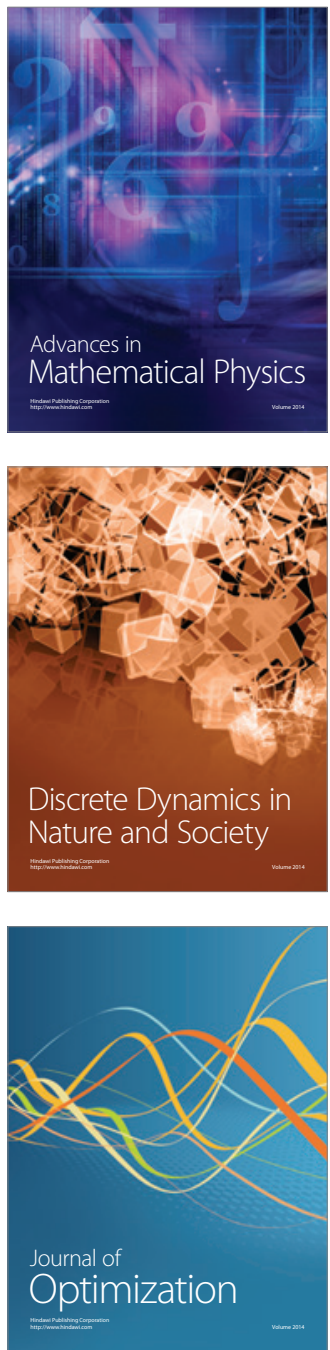